\title{
Molecular and serological dynamics of Chlamydia pecorum infection in a longitudinal study of prime lamb production
}

\author{
Sankhya Bommana $^{1}$, Evelyn Walker ${ }^{2}$, Marion Desclozeaux ${ }^{1}$, Martina Jelocnik ${ }^{1}$, Peter Timms $^{1}{ }^{\text {, }}$ Adam \\ Polkinghorne $^{1}$, Scott Carver ${ }^{\text {Corresp. }}{ }^{3}$ \\ ${ }^{1}$ Centre for Animal Health Innovation, University of the Sunshine Coast, Sippy Downs, Australia \\ 2 Central West Local Land Services, Dubbo, Australia \\ 3 School of Biological Sciences, University of Tasmania, Hobart, Tasmania, Australia \\ Corresponding Author: Scott Carver \\ Email address: scott.carver@utas.edu.au
}

Background. Chlamydia pecorum is a globally significant livestock pathogen causing pathology and production losses. The on-farm infection and serological dynamics and the relevance of existing diagnostic tools for diagnosing C. pecorum in livestock remains poorly characterized. In this study, we characterized the antigen and antibody dynamics of this pathogen in a longitudinal study of prime lamb production, utilizing the infection focused $C$. pecorum-specific 16S rRNA qPCR assay and serology based chlamydial Complement fixation Test (CFT).

Methods. The study consisted of 76 Border Leicester mixed sex lambs (39 females and 37 males) that were sampled bimonthly from 2-10 months of age in a commercial farm operating in Central NSW, Australia. Blood/plasma was analysed for CFT antibodies, and swabs from conjunctival, rectal and vaginal sites were analysed for $C$. pecorum shedding using qPCR. We assessed the temporal and overall dynamics of $C$. pecorum in lambs, including detailed description and comparison of qPCR and CFT, the timing of first detection by either diagnostic method, the lag between infection and antibody response; and the distribution of qPCR load and CFT antibody titre over time.

Results. Over the study period, C. pecorum was highly prevalent ( $71.0 \%$ by qPCR, $92.1 \%$ by CFT, $96.0 \%$ by both), with $21.1 \%$ (16/76) lambs shedding $\geq 1000$ qPCR copies/ $\mu$ (denoted as high shedders). $C$. pecorum shedding (as evidence of infection) were first observed at 2 months of age (14.4\%) with a significant peak of infection occurring at 6 months of age (34.2\%), whereas seroconversions peaked at 8 months of age (81.5\%). 52.6\% of $C$. pecorum qPCR and CFT positive lambs became qPCR negative by 10 months of age, indicating clearance of chlamydial infection. Although CFT is utilised for on-farm detection of active infection, we confirm that it lagged behind qPCR detection (average lag $1.7 \pm 2.1$ months) and that the proportion of qPCR positives simultaneously identified by CFT was low with 2/11 (18.1\%), 0/13, $17 / 25(68.0 \%), 5 / 7(71.4 \%)$ and $1 / 10$ (10.0\%) concurrent seroconversions occurring at 2, 4, 6, 8 and 10 months of age, respectively.

Discussion. This work reveals rapid rates of $C$. pecorum infection and widespread exposure during lamb production. The comparison of molecular and serological diagnostic agreement longitudinally, supports the use of qPCR as an important ancillary tool for the detection of active infections in conjunction with chlamydial CFT for routine veterinary diagnostics. Development of rapid Point-of-Care (POC) tools for diagnosing active infection would be valuable for producers and veterinarians. 


\section{Molecular and serological dynamics of Chlamydia pecorum}

\section{2 infection in a longitudinal study of prime lamb production}

4 Sankhya Bommana ${ }^{1}$, Evelyn Walker ${ }^{1,2}$, Marion Desclozeaux ${ }^{1}$, Martina Jelocnik ${ }^{1}$, Peter Timms ${ }^{1}$,

5 Adam Polkinghorne ${ }^{1}$ and Scott Carver $^{3}$

$6{ }^{1}$ Centre for Animal Health Innovation, University of the Sunshine Coast, Sippy Downs,

7 Australia

$8 \quad{ }^{2}$ Central West Local Land Services, Dubbo, Australia

$9{ }^{3}$ School of Biological Sciences, University of Tasmania, Hobart, Australia

11 Corresponding Author:

12 Scott Carver $^{3}$

13 School of Biological Sciences, University of Tasmania, Hobart, 7005, Australia

14 Email address: scott.carver@utas.edu.au (S Carver) 


\section{Abstract}

27 Background. Chlamydia pecorum is a globally significant livestock pathogen causing pathology and production losses. The on-farm infection and serological dynamics and the relevance of existing diagnostic tools for diagnosing C. pecorum in livestock remains poorly characterized. In this study, we characterized the antigen and antibody dynamics of this pathogen in a longitudinal study of prime lamb production, utilizing the infection focused C. pecorum-specific 16S rRNA qPCR assay and serology based chlamydial Complement fixation Test (CFT).

Methods. The study consisted of 76 Border Leicester mixed sex lambs (39 females and 37 males) that were sampled bimonthly from 2-10 months of age in a commercial farm operating in Central NSW, Australia. Blood/plasma was analysed for CFT antibodies, and swabs from conjunctival, rectal and vaginal sites were analysed for $C$. pecorum shedding using qPCR. We assessed the temporal and overall dynamics of $C$. pecorum in lambs, including detailed description and comparison of qPCR and CFT, the timing of first detection by either diagnostic method, the lag between infection and antibody response; and the distribution of qPCR load and CFT antibody titre over time.

41 Results. Over the study period, C. pecorum was highly prevalent $(71.0 \%$ by qPCR, $92.1 \%$ by

42 CFT, $96.0 \%$ by both), with $21.1 \%$ (16/76) lambs shedding $\geqslant 1000 \mathrm{qPCR}$ copies/ $\mu$ l (denoted as high shedders). C. pecorum shedding (as evidence of infection) were first observed at 2 months

44 of age (14.4\%) with a significant peak of infection occurring at 6 months of age $(34.2 \%)$, 45 whereas seroconversions peaked at 8 months of age (81.5\%). 52.6\% of C. pecorum qPCR and

46 CFT positive lambs became qPCR negative by 10 months of age, indicating clearance of 
47 chlamydial infection. Although CFT is utilised for on-farm detection of active infection, we

48 confirm that it lagged behind qPCR detection (average lag $1.7 \pm 2.1$ months) and that the

49 proportion of qPCR positives simultaneously identified by CFT was low with 2/11 (18.1\%), 0/13,

$5017 / 25(68.0 \%), 5 / 7(71.4 \%)$ and $1 / 10(10.0 \%)$ concurrent seroconversions occurring at 2, 4, 6, 8

51 and 10 months of age, respectively.

52 Discussion. This work reveals rapid rates of $C$. pecorum infection and widespread exposure

53 during lamb production. The comparison of molecular and serological diagnostic agreement

54 longitudinally, supports the use of qPCR as an important ancillary tool for the detection of active

55 infections in conjunction with chlamydial CFT for routine veterinary diagnostics. Development

56 of rapid Point-of-Care (POC) tools for diagnosing active infection would be valuable for

57 producers and veterinarians.

\section{Introduction}

60

Despite growing evidence that infections of the obligate intracellular bacterial pathogen, Chlamydia pecorum, are ubiquitous among some of the most economically important livestock species globally, the epidemiology and pathologies associated with this pathogen are poorly understood (Walker et al. 2015). C. pecorum infections of sheep, cattle and goats are associated with polyarthritis (Walker at al. 2016), keratoconjunctivitis (Polkinghorne et al. 2009), sporadic bovine encephalomyelitis (SBE) and pneumonia (Jelocnik et al. 2014a). Sporadic cases of ovine and caprine abortions due to C. pecorum have also been reported (Giannitti et al. 2016; Walker et al. 2015). C. pecorum chlamydiosis in both sheep and cattle can limit growth and survival of young rapidly growing stock and, such weight loss or failure to thrive as a result of $C$. pecorum polyarthritis, is the primary economic concern for farmers (Poudel et al. 2012; Walker et al. 2015; 
70 Walker et al. 2016). The latter disease is a compelling one for Australian producers with $2.1 \%$ of

71 lambs and $1.6 \%$ of calves condemned each year at Australian abattoirs as a result of polyarthritis,

72 estimated to cost the livestock industry around $\$ 30 \mathrm{M}$ annually (Walker et al. 2016). Similar

73 economic costs of arthritis have also been reported elsewhere (Dupuy et al. 2013). The specific

74 contribution of arthritis-associated losses by C. pecorum are yet to be established, however.

75 The complex relationship between C. pecorum infection and overt animal pathology makes the

76 diagnosis and control of infections challenging. Sub-clinical, asymptomatic infections are

77 common, characterised by the detection of C. pecorum in the faeces, gastrointestinal and/or

78 urogenital tract of so-called "shedder" animals (Reinhold et al. 2008; Reinhold et al. 2011).

79 These same animals may also act as an important reservoir, facilitating infection of individuals

80 who exhibit symptomatic infections. Indeed, in Australia, the largest exporter of sheep globally,

81 C. pecorum was recently estimated to be present in $30 \%$ of the country's sheep flock, based on

82 faecal shedding alone (Yang et al. 2016). While these infections are common, it is apparent that

83 C. pecorum can also disseminate to other tissues where it can replicate in epithelial cells and

84 macrophages of the conjunctival, genital and intestinal sites, in synoviocytes of the joint tissue

85 and occasionally, the respiratory tract (Jelocnik et al. 2014a; Twomey et al. 2006). The factors

86 that influence dissemination and pathogenesis of these strains are currently unknown, although

87 molecular typing studies have suggested that genetic differences may exist between strains

88 associated with disease and those found asymptomatically colonising the gastrointestinal tract

89 (Jelocnik et al. 2013; Jelocnik et al. 2014b; Mohamad et al. 2014).

90 In Australia, diagnosis of sheep chlamydiosis is based on clinical history, symptoms and

91 presenting pathology, and is routinely confirmed by diagnostic laboratories using a Complement

92 Fixation Test (CFT). Chlamydial CFT detects antibodies (Ab) to either whole chlamydial 
93 elementary bodies (EBs) or Chlamydiaceae-specific lipopolysaccharide and remains as the

94 recommended test for Chlamydia diagnosis by the World Organisation for Animal Health and

95 Sub-Committee for Animal Health Laboratory Standards (Sachse et al. 2009). The use of crude

96 or partially purified antigen in CFT depends on the binding of anti-Chlamydiaceae antibodies of

97 the host species to guinea pig complement, and has highly variable sensitivity depending on the

98 host species and antibody isotype (Kaltenboeck et al. 1997; Perez-Martinez et al. 1986).

99 Moreover, use of whole chlamydial elementary bodies (EBs) or Chlamydiaceae-specific

100 lipopolysaccharide as an antigen in this assay renders only genus-specificity and inevitable

101 serological cross-reactivity with Chlamydia-related organisms and gram negative bacteria

102 (Casson et al. 2007; Haralambieva et al. 2001). Detection of sero-conversion by CFT has

103 multiple purposes such as: (a) confirmation of chlamydiosis; (b) the presence or absence of

104 chlamydial infection; and (c) determination of immune status after vaccination (Sachse et al.

105 2009). The major issue is that CFT has a largely unknown relationship to either the acute,

106 convalescent or persistent phase of C. pecorum infection or even pathology itself (Griffiths et al.

107 1996; Kaltenboeck et al. 1997; Perez-Martinez et al. 1986). Alongside this unknown relationship,

108 C. pecorum shedding (measured by qPCR) at the gastrointestinal tract has not well correlation to

109 disease or pathology and has not been investigated further to date (Walker et al. 2016).

110 Beyond the use of CFT and qPCR for the diagnosis of C. pecorum cell culture or embryonated

111 hens' egg based isolation traditional methods exist that are often cumbersome and time

112 consuming for on-farm settings (Sachse et al. 2009). Here we conducted a longitudinal study of

113 C. pecorum infection in a prime lamb flock. Our aims were to: (a) describe the temporal and

114 overall dynamics of $C$. pecorum in lambs, including detailed description and comparison of

115 qPCR and CFT, the timing of first detection by either diagnostic method, and estimate the lag 
116 between infection (qPCR) and antibody response (CFT); and (2) detail the distribution of

117 chlamydial qPCR load and CFT antibody titre over time. For both aims, we detail consistency

118 and variation in infection across three anatomical sampling sites: conjunctiva, rectum and vagina.

119

120 Materials and Methods

121 Lamb husbandry. This study followed 76 Border Leicester mixed sex lambs operating in a 122 commercial sheep farm in Central Western NSW. Lambs in this study were managed as per

123 normal farm husbandry practices (e.g. marking, feeding, weaning, vaccinations etc.). At time of

124 marking (two months of age), the lambs were uniquely ear tagged and sampled at bi-monthly

125 intervals (2, 4, 6, 8 and 10 months) until finishing which is at the 10 months of age.

126 Blood and swab samples collection. Swab samples taken from conjunctiva, rectum and vagina, 127 and blood samples were collected from individual lambs across five bimonthly sampling time 128 points from 2-10 months of age. Collected swabs were used for in-house DNA amplification 129 assays, and the blood samples were submitted to the State Veterinary Diagnostic Lab, Elizabeth

130 Macarthur Agricultural Institute, Menangle, NSW, for serological testing by Chlamydia CFT. A

131 serum sample was considered positive by CFT with a titer of 16 or greater in this study. The

132 collection and testing of these swabs and blood samples was approved by the University of the

133 Sunshine Coast Animal Ethics Committee (AN/S/14/31).

134 Swab processing and DNA extraction. Clinical swabs were processed according to the in-

135 house swab processing methods (Jelocnik et al. 2013). Briefly, swabs were dispensed into $1.5 \mathrm{ml}$

136 of sucrose-phosphate-glutamate buffer by vortexing and centrifugation. The resulting cell pellet

137 was resuspended in tris-EDTA buffer and heated at $95 \mathrm{C}$ for 10 minutes to heat-inactivate the

138 elementary bodies (EBs) for further processing at room temperature. For all swabs, DNA was 
139 extracted using a QIAamp DNA kit (Qiagen, Doncaster, Victoria, Australia), according to the

140 manufacturer's instructions. DNA purity and yield was determined using a NanoDrop

141 spectrophotometer ND-1000 (Thermo Fisher Scientific, Inc.).

142 C. pecorum 16S species- specific qPCR screening. Conjunctival, vaginal and rectal swabs

143 were screened for the presence of C. pecorum infections by a C. pecorum-specific quantitative

144 PCR targeting the 16S rRNA gene modified from previously published protocol (Marsh et al.

145 2011). The C. pecorum 16S 204 bp fragment (RT-Cpec -F: 5'-

146 AGTCGAACGGAATAATGGCT-3', RT-Cpec-R: 5'-CCAACAAGCTGATATCCCAC-3'; IDT)

147 was sub-cloned into pGem-T Easy (Promega) and amplified with M13 universal primers to

148 generate a M13-Cpec-16S fragment. Serial dilutions of the M13-Cpec-16S fragment were used

149 to produce a standard curve by mixing $5 \mu 1$ of diluted fragment with RT-Cpec-F and RT-Cpec-R

150 primers $\left(1 \mu \mathrm{M}\right.$ final) and $1 \mathrm{X}$ QuantiTect SYBR ${ }^{\circledR}$ Green PCR mix (Qiagen) in a final volume

151 reaction of $20 \mu \mathrm{l}$. Cycling conditions were $95^{\circ} \mathrm{C}-15 \mathrm{~min}$, followed by 35 cycles of $94^{\circ} \mathrm{C}-15 \mathrm{sec}$,

$15257^{\circ} \mathrm{C}-15 \mathrm{sec}, 72^{\circ} \mathrm{C}-30 \mathrm{sec}$, and a final amplification cycle of $72^{\circ} \mathrm{C}, 10 \mathrm{~min}$. Diluted C. pecorum

153 strain MC/Marsbar served as a positive control while $\mathrm{dH}_{2} \mathrm{O}$ was used as negative control. All

154 samples were tested in duplicates. The limit of detection load was 10 C. pecorum 16S rRNA

155 copies/ $\mu 1$.

156

157 Statistical analysis

158 We undertook a detailed individual assessment of the timing and diagnostic characteristics of $C$.

159 pecorum infection dynamics. This included the diagnostic infection and serological response

160 (qPCR and CFT) status at each sampling time point (Table 1), information on evidence of

161 individual clearance of infection (as determined by a lamb having both a qPCR and CFT 
162 diagnosis across the sampling time points, but only being CFT positive by 10 months of age).

163 This individual level information facilitated flock-wide description of C. pecorum infection

164 dynamics and diagnostic evaluations, including overall and time dependent CFT and qPCR

165 positivity and the diagnostic states (qPCR+CFT+, qPCR+CFT-, qPCR-CFT+, qPCR-CFT-)

166 (Table 2). We evaluated the 'qPCR CFT agreement' as the percentage of qPCR positive and

167 negative lambs with matching CFT diagnosis

168

$$
\frac{(a+d)}{(a+b+c+d)} \times 100
$$

169

where $a$ is diagnostic state $\mathrm{qPCR}+\mathrm{CFT}+$, $\mathrm{b}$ is $\mathrm{qPCR}+\mathrm{CFT}-\mathrm{c}$ is qPCR-CFT+, and $\mathrm{d}$ is qPCR-

170 CFT-. We have specifically avoided the widely used 'sensitivity' and 'specificity' terminology

171 owing to the knowledge that qPCR and CFT are measuring inherently different things (bacterial

172 agent and antibodies, respectively). The comparison of CFT against qPCR is nevertheless

173 functional on applied grounds, owing to its relatively common use in veterinary settings to

174 diagnose active infection.

175 Focussing only on the month at which first qPCR diagnosis was made, individual lags of CFT

176 from qPCR diagnosis was made followed by average \pm standard deviation to get the

177 population/flock lag. Lag in CFT from qPCR was calculated on the basis of animals that had

178 their qPCR detection first followed by/concurrent CFT detection $(n=45)$ (Tables S1-S3). Lambs

179 that had CFT detection arise before $\mathrm{qPCR}(\mathrm{n}=31)$ as a result of previous infections that were

180 missed due to bimonthly sampling frequency were not included in the lag analyses.

181 All data analyses was performed in Microsoft Excel 2013 and graphs outputs (Fig 1 and 2)

182 generated using GraphPad Prism version 7 (GraphPad Software, LaJolla, CA, USA).

\section{Results}


185 Temporal and overall dynamics of $C$. pecorum exposure in lambs.

186 Diagnosis of $C$. pecorum infections revealed an overall positivity of $71.0 \%$ by qPCR, $92.1 \%$ by

187 CFT and $96.0 \%$ by either test over the study period (Table 1 ). The temporal patterns of qPCR

188 detection were broadly consistent among the samples from the conjunctiva, rectum and vagina,

189 with greatest number of detection at 6 months of age and similar among other months of

190 sampling (Table 1). While temporal patterns in qPCR were similar among sampling sites, greater

191 numbers of detection were made from the conjunctiva, relative to the rectum and vagina (Table

192 1). Detection of $C$. pecorum by CFT rose rapidly from 6 months of age, peaking at 8 months of

193 age and then declining by 10 months of age (Table 1). A notable number of CFT detection $(\mathrm{n}=10)$

194 were made at 2 months of age, and possibly indicate the presence of maternal antibodies, or

195 infections that occurred and resolved $<2$ months of age. Temporal agreement between qPCR and

196 CFT was greatest at 2 months of age, decreased to lowest levels at 8 months of age (owing to an

197 increased number of lambs that were positive by CFT, but negative by qPCR), and increased

198 again by 10 months of age (owing to an increased number of lambs negative by both diagnostic

199 methods) (Table 2). Approximately half of the lambs (52.6\%; 40/76) sampled exhibited evidence

200 of infection clearance (only CFT detection by 10 months of age) within the sampling frame of

201 this study (Table 2)

202 In this study, 67.1\% of lambs (51/76) tested positive by both CFT and qPCR (Table 2). However,

203 3/51 of these lambs had CFT antibodies arise before qPCR detection due to past infection that

204 went undiagnosed as a result of bimonthly sampling or detection of maternal antibodies at 2

205 months of age (Table S1). Interestingly, 3.9\% (3/76) of lambs were qPCR positive and CFT

206 negative throughout the study period and $2 / 3$ of these lambs had recurrent $C$. pecorum infection

207 as measured by repeated qPCR positivity (Table 2$)$. We identified a further $25 \%(19 / 76)$ of 
208 lambs that tested positive by CFT and negative by qPCR during the entire study period of 2 to 10 209 months of age, and 3.9\% (3/76) of lambs tested qPCR and CFT negative during the entire study

210 period (Table 2).

211 We also investigated the timing of first detection by qPCR and CFT to gain an improved

212 understanding of when lambs first became infected and seroconverted (Table 3 and 4). Peaks in

213 timing of first detection by qPCR and CFT were 6 and 8 months of age, respectively (Table 3

214 and 4), which was broadly similar to what was observed when evaluating the flock as a whole

215 (Table 1). Most of the first seroconversions (CFT diagnoses) were detected concurrently with

216 first detection of infection, a smaller number lagged behind infection and a moderate number

217 occurred without detection of infection, which may indicate the 'window of infection' that was

218 missed due to the bi-monthly sampling intervals (Table 3 and 4). Overall, the sampling intervals

219 in this study led to an estimated average time lag between first qPCR detection and first CFT

220 detection of $1.7 \pm 2.1$ months.

221 Distribution of $C$. pecorum bacterial load and antibody titre in lambs.

222 C. pecorum bacterial load (qPCR copy number) and CFT antibody titer exhibited distinct

223 temporal patterns (Table 5, Fig 1a and b). From 2-6 months of age, lambs exhibited an overall

224 increase in chlamydial loads, peaking at 6 months of age (Table 5, Fig 1a). Across all time points, 225 a small number of lambs had loads exceeding 1000 16S rRNA gene copy numbers (Fig 1a, data 226 points in red). The temporal patterns in bacterial load varied among the sampling sites (Fig 2). In

227 the conjunctiva, loads trended downward from 2-10 months of age (Fig 2a). In contrast, bacterial

228 loads increased from 2-6 months of age at the rectal sampling site, diminishing markedly

229 thereafter (Fig 2b). For female lambs the greatest loads occurred from 6-10 months of age at the

230 vaginal sampling site (Fig 2c). Overall, 16/76 (21\%) of lambs were shedding high loads of $C$. 
231 pecorum DNA ( $\geq 1000$ copies/ $\mu$ l) from their mucosal sites of conjunctiva (3/76), rectum $(4 / 76)$

232 and vagina (9/76), respectively (Fig 2). CFT titres were low at 2-4 months of age, and then

233 peaked from 6-8 months of age, followed by a slight decline in titre at 10 months of age (Table 5,

234 Fig 1b).

235

236 Discussion

237 Despite the significant economic loss in livestock due to chlamydiosis, C. pecorum infections

238 continue to be under recognized as a major endemic pathogen for producers globally (Walker et

239 al. 2015; Walker et al. 2016). Key factors contributing to this underestimation are the lack of

240 appreciation of infection dynamics, relative to antibody dynamics (Berri et al. 2009; Sachse et al.

241 2009). To address these issues, the objective of our study was to describe infection (measured by

242 a species specific 16S qPCR assay) and serological (measured by CFT) dynamics using a

243 longitudinal on-farm sheep flock setting in Central NSW, Australia.

244 Whether detected by qPCR, CFT or both diagnostic methods, the overwhelming majority of

245 lambs were exposed to C. pecorum at some point over the study period, indicating rapid rates of

246 infection, and widespread exposure in the flock. Based on the sampling frequency employed, we

247 observed that lambs experienced peak chlamydial infections over a period of 2-6 months of age

248 followed by seroconversion at 6-8 months of age, and occasional detection of low-level recurrent

249 chlamydial infection at 10 months. The sudden rise in infection prevalence at six months

250 potentially reflects a response to the stress of marking, and associated herding of lambs into

251 yards, increasing contact between infected lambs, and precipitating spread of chlamydial

252 infections (Poudel et al. 2012; Stanley \& Jones 2003). Marking or mulesing has been recorded as

253 the peak time for risk of infections by Erysipelas, the other major cause of bacterial arthritis in 
254 lambs (Robson 2003). Peak seroconversion of lambs occurred at eight months of age, although a

255 subset of lambs had circulating antibodies as early as two months, likely owing to maternal

256 transfer of antibodies or early seroconversion in cases that were also qPCR positive. At early

257 time points ( 2 and 4 months), qPCR preceded CFT in detecting new infections, with a peak in

258 coincidental first detection by both methods occurring at six months. Based on these findings,

259 qPCR performs well at detecting infections early in life (2-6 months), new infections, and

260 potentially recurrent infections. Antibody responses appeared to confer a degree of protection, as

261 indicated by animals becoming qPCR negative in 52.6\% (40/76) of cases by 10 months.

262 However, our results indicated that the protection was not long lasting, as both declining

263 seroprevalence, waning titres from 8-10 months of age and absence of CFT titres in some cases

264 was observed, and may have contributed to repeat infections detected during these months $(\mathrm{n}=8$

265 and $\mathrm{n}=6$ at 8 and 10 months, respectively).

266 CFT is periodically utilized for diagnosis of active C. pecorum infection in veterinary settings,

267 hence it was important to characterize its association to qPCR diagnosis. We found that using

268 CFT potentially obscures the ability to detect acute active chlamydial infections with 9/11

269 (81.8\%), 13/13 (100.0\%), 8/25 (32.0\%), 2/7 (28.5\%) and 9/10 (90.0\%) qPCR detection

270 preceding seroconversion at 2, 4, 6, 8 and 10 months of age, respectively. This important finding

271 of poor matching between CFT and qPCR is similar to results reported in other Chlamydia

272 studies (Bas et al. 2001; McCauley et al. 2007; Persson \& Boman 2000; Sachse et al. 2009).

273 Overall, our results show that CFT has limited value for diagnosis of acute active infections. In

274 this study, we also identified three lambs that were qPCR positive (2/3 lambs were repeatedly

275 qPCR positive) but had no detectable CFT antibodies longitudinally. In our previous study on

276 characterisation of humoral immune responses to naturally occurring C. pecorum infections we 
277 have identified moderate antigen specific MOMP and PmpG IgG antibodies present in these

278 three lambs longitudinally (Bommana et al. 2017). These findings elude us to believe that these

279 animals are not passive shedders of C. pecorum and that in reality CFT as an assay has relatively

280 poor sensitivity. CFT has also been criticized for its requirement of technical expertise,

281 subjective interpretation, and its unsuitability for testing large numbers of specimens; when

282 testing C. abortus infections in sheep (McCauley et al. 2007; McCauley et al. 2010). Conversely,

283 our species-specific qPCR assay appears a much improved tool for early and active stages of

284 infection (Sachse et al. 2009). CFT as a diagnostic assay is suitable for seroprevalence surveys

285 and serological dynamic studies (such as this study) as it can detect antibodies to clinically

286 unapparent chlamydial infections. CFT is inappropriate for the retrospective diagnosis of

287 chlamydial mucosal infections, such as in oculo-genital sites that make antibodies specific to a

288 localised site of infection (Barnes 1989; Griffiths et al. 1996). The exception to this is diagnosis

289 of chlamydial polyarthritis or abortion in ruminants, wherein high exposure to Chlamydia elicits

290 a pronounced systemic increase in antibody levels (Perez-Martinez et al. 1986; Bommana et al.

291 2017). Potentially, there may exist early seroconversion or antibody markers for improved

292 serological diagnosis of acute-phase infection in livestock, overcoming the shortcomings of CFT.

293 Future research is needed to identify such markers by investigating proteomic profiles of

294 chlamydial infections to differentiate responses related to acute infections, cured past infection,

295 and persistent infection. Improved diagnostic tools for acute phase infection, or exposure, that

296 are simple to undertake and interpret, would be of significant value for routine use by

297 veterinarians and veterinary diagnostic laboratories (Jelocnik et al. 2017).

298 A notable proportion $(21.1 \% ; 16 / 76)$ of the flock were shedding high numbers $\left(>1 \times 10^{4}-10^{5}\right)$ of

299 C. pecorum, which may have important implications for C. pecorum dynamics. "Supershedders" 
300 are suspected to facilitate transmission of many pathogens (Lloyd-Smith et al. 2005; Matthews et

301 al. 2013). In this study lambs shedding high amounts of $C$. pecorum may facilitate transmission

302 through fecal-oral contact or greater environmental contamination (eg. water troughs, grazing

303 pasture) (Stanley \& Jones 2003). Rapid POC diagnostic tools underway for C. pecorum and

304 C.psittaci, (Jelocnik et al. 2017) are needed to identify these individuals as part of a preventative

305 flock management program. Any animal that is identified early as a supershedder could be culled 306 to avoid further flock transmission and reduce infection rates. More broadly this observation fits

307 with a body of literature suggesting that a small proportion of individuals are commonly

308 responsible for the majority of transmission events (the 80:20 rule for supershedders and

309 superspreaders) across multiple systems (May \& Anderson 1987; Woolhouse et al. 1997). One

310 similar example was recorded in the livestock industry when faecal sample analysis conducted at

311 a UK abattoir revealed that approximately $9 \%$ of the cattle examined over a 9-week period were

312 high Escherichia coli $\mathrm{O} 157$ shedders ( $\left.>10^{4} \mathrm{CFU} / \mathrm{g}\right)$ and they accounted for over $96 \%$ of the

313 bacteria shed by all animals tested (Omisakin et al. 2003).

\section{Conclusion}

316 In this study, we have described the on-farm molecular and serological dynamics of the

317 enigmatic pathogen, C. pecorum in lambs from 2 to 10 months of age. Lambs exhibiting $C$.

318 pecorum infections had high rates of infection at 6 months of age and seroconversion at 8 months

319 of age. Based on our individual diagnostic assessments of qPCR and CFT, C. pecorum-specific

320 qPCR was found to be a more useful tool in diagnosing acute active infections early on in life (2-

3216 months), recurring infections, and also in detecting C. pecorum positivity in CFT negative

322 animals. Further integration of the findings in this study with detailed pathological investigations 
323 in relation to chlamydial shedding, disease and impact are currently underway. Findings in this

324 study provide insights into the infection and antibody dynamics, and ways to improve diagnosis

325 of C. pecorum infections in sheep. While CFT is the primary assay in diagnosing chlamydial

326 infections of livestock in veterinary settings, C. pecorum-specific qPCR assay will serve as an

327 important ancillary tool when used in parallel with CFT in diagnosing active acute on farm

328 infections of this pathogen. Further development of rapid POC assays for routine veterinary

329 diagnostic testing of C. pecorum infections in Australian livestock would be valuable (Jelocnik et

330 al. 2017). This is particularly the case for clinical cases with variable presentations, such as

331 conjunctivitis, asymptomatic infections and/or conjunctivitis with polyarthritis. Considering the

332 ever increasing availability of species and strain specific qPCR assays for member species in the

333 genus Chlamydia, sensitive discriminatory assays for C. pecorum would be beneficial for both

334 producers and researchers (Jelocnik et al. 2017; Sachse et al. 2009). Such tools will be essential

335 if we are to truly understand the true prevalence and impact of these infections on livestock

336 production globally.

\section{Acknowledgements}

339 We would like to acknowledge Central West Local Land Services District Veterinarians and

340 Biosecurity Officers for their on farm assistance in this project. 


\section{References}

344 Barnes RC. 1989. Laboratory diagnosis of human chlamydial infections. Clinical Microbiology

$345 \quad$ Reviews 2:119-136.

346 Bas S, Muzzin P, Ninet B, Bornand JE, Scieux C, and Vischer TL. 2001. Chlamydial serology: comparative diagnostic value of immunoblotting, microimmunofluorescence test, and immunoassays using different recombinant proteins as antigens. Journal of Clinical

Berri M, Rekiki A, Boumedine K, and Rodolakis A. 2009. Simultaneous differential detection of Chlamydophila abortus, Chlamydophila pecorum and Coxiella burnetii from aborted ruminant's clinical samples using multiplex PCR. BMC Microbiology 9:130. 10.1186/1471-2180-9-130

Bommana S, Walker E, Desclozeaux M, Timms P, Polkinghorne A. 2017. Humoral immune response against two surface antigens of Chlamydia pecorum in vaccinated and naturally infected sheep. PLoS ONE 12(11): e0188370.

Casson N, Entenza JM, Greub G. 2007. Serological cross-reactivity between different Chlamydia-like organisms. Journal of Clinical Microbiology 45(1):234-6.

Giannitti F, Anderson M, Miller M, Rowe J, Sverlow K, Vasquez M, Canton G. 2016. Chlamydia pecorum: fetal and placental lesions in sporadic caprine abortion. Journal of Veterinary Diagnostic Investigation 28(2):184-9. 
366 Griffiths PC, Plater JM, Horigan MW, Rose MP, Venables C, and Dawson M. 1996.

367 Serological diagnosis of ovine enzootic abortion by comparative inclusion

368 immunofluorescence assay, recombinant lipopolysaccharide enzyme-linked

369 immunosorbent assay, and complement fixation test. Journal of Clinical

$370 \quad$ Microbiology 34:1512-1518.

371 Haralambieva I, Iankov I, Petrov D, Ivanova R, Kamarinchev B, Mitov I. 2001. Cross-

372 reaction between the genus-specific lipopolysaccharide antigen of Chlamydia spp. and

373 the lipopolysaccharides of Porphyromonas gingivalis, Escherichia coli 0119

374 and Salmonella newington: implications for diagnosis. Diagnostic Microbiology and

375 Infectious Disease 41(3):99-106.

376 Jelocnik M, Forshaw D, Cotter J, Roberts D, Timms P, and Polkinghorne A. 2014a. Molecular and pathological insights into Chlamydia pecorum-associated sporadic bovine encephalomyelitis (SBE) in Western Australia. BMC Veterinary Research 10:1-9. $10.1186 / 1746-6148-10-121$

380

381

382

383

384

385

386

387

388

389

Jelocnik M, Frentiu FD, Timms P, and Polkinghorne A. 2013. Multi-locus sequence analysis provides insights into the molecular epidemiology of Chlamydia pecorum infections in Australian sheep, cattle and koalas. Journal of Clinical Microbiology 51:2625-2632. $10.1128 / \mathrm{jcm} .00992-13$

Jelocnik M, Islam MM, Madden D, Jenkins C, Branley J, Carver S, and Polkinghorne A. 2017. Development and evaluation of rapid novel isothermal amplification assays for important veterinary pathogens: Chlamydia psittaci and Chlamydia pecorum. PeerJ 5:e3799. 10.7717/peerj.3799

Jelocnik M, Walker E, Pannekoek Y, Ellem J, Timms P, and Polkinghorne A. 2014b. Evaluation of the relationship between Chlamydia pecorum sequence types and disease using a 
392 Kaltenboeck B, Heard D, DeGraves FJ, and Schmeer N. 1997. Use of synthetic antigens improves detection by enzyme-linked immunosorbent assay of antibodies against abortigenic Chlamydia psittaci in ruminants. Journal of Clinical Microbiology 35:22932298.

Lloyd-Smith JO, Schreiber SJ, Kopp PE, and Getz WM. 2005. Superspreading and the effect of individual variation on disease emergence. Nature 438:355-359. 10.1038/nature04153

Marsh J, Kollipara A, Timms P, and Polkinghorne A. 2011. Novel molecular markers of Chlamydia pecorum genetic diversity in the koala (Phascolarctos cinereus). BMC Microbiology 11:77. 10.1186/1471-2180-11-77

401 Matthews L, Reeve R, Gally DL, Low JC, Woolhouse MEJ, McAteer SP, Locking ME, Chase402 Topping ME, Haydon DT, Allison LJ, Hanson MF, Gunn GJ, and Reid SWJ. 2013.

May RM, and Anderson RM. 1987. Transmission dynamics of HIV infection. Nature 326:137407 Predicting the public health benefit of vaccinating cattle against Escherichia coli O157.

McCauley LM, Lancaster MJ, Young P, Butler KL, and Ainsworth CG. 2007. Comparison of ELISA and CFT assays for Chlamydophila abortus antibodies in ovine sera. Australian Veterinary Journal 85:325-328. 10.1111/j.1751-0813.2007.00189.x

411 McCauley LME, Lancaster MJ, Butler KL, and Ainsworth CGV. 2010. Serological analysis of Chlamydophila abortus in Australian sheep and implications for the rejection of breeder 

0813.2009.00536.x

415 Mohamad KY, Kaltenboeck B, Rahman KS, Magnino S, Sachse K, and Rodolakis A. 2014. Host adaptation of Chlamydia pecorum towards low virulence evident in co-evolution of the

Omisakin F, MacRae M, Ogden ID, and Strachan NJ. 2003. Concentration and prevalence of ompA, incA, and ORF663 Loci. PLoS ONE 9:e103615. 10.1371/journal.pone.0103615 Escherichia coli $\mathrm{O} 157$ in cattle feces at slaughter. Applied and Environmental Microbiology 69:2444-2447.

Perez-Martinez JA, Schmeer N, and Storz J. 1986. Bovine chlamydial abortion: serodiagnosis by modified complement-fixation and indirect inclusion fluorescence tests and enzymelinked immunosorbent assay. American Journal of Veterinary Research 47:1501-1506.

Persson K, and Boman J. 2000. Comparison of five serologic tests for diagnosis of acute infections by Chlamydia pneumoniae. Clinical and Diagnostic Laboratory Immunology 7:739-744.

Polkinghorne A, Borel N, Becker A, Lu ZH, Zimmermann DR, Brugnera E, Pospischil A, Vaughan L. 2008. Molecular evidence for chlamydial infections in the eyes of sheep. Veterinary Microbiology. 135(1-2):142-6.

Poudel A, Elsasser TH, Rahman Kh S, Chowdhury EU, and Kaltenboeck B. 2012. Asymptomatic endemic Chlamydia pecorum infections reduce growth rates in calves by up to 48 percent. PLoS ONE 7. 10.1371/journal.pone.0044961

433 Reinhold P, Jaeger J, Liebler-Tenorio E, Berndt A, Bachmann R, Schubert E, Melzer F, Elschner M, and Sachse K. 2008. Impact of latent infections with Chlamydophila species in young cattle. Veterinary Journal 175. 10.1016/j.tvj1.2007.01.004 
436 Reinhold P, Sachse K, and Kaltenboeck B. 2011. Chlamydiaceae in cattle: commensals, trigger 437 organisms, or pathogens? Veterinary Journal 189. 10.1016/j.tvj1.2010.09.003

438 Robson S. 2003. Bacterial arthritis in lambs: NSW Agriculture.

439 Sachse K, Vretou E, Livingstone M, Borel N, Pospischil A, and Longbottom D. 2009. Recent

440 developments in the laboratory diagnosis of chlamydial infections. Veterinary

$441 \quad$ Microbiology 135:2-21. http://dx.doi.org/10.1016/j.vetmic.2008.09.040

442 Stanley K, and Jones K. 2003. Cattle and sheep farms as reservoirs of Campylobacter. Journal of Applied Microbiology 94:104-113. 10.1046/j.1365-2672.94.s1.12.x

444 Twomey DF, Griffiths PC, Horigan MW, Hignett BC, and Martin TP. 2006. An investigation 445 into the role of Chlamydophila spp. in bovine upper respiratory tract disease. Veterinary

447 Walker E, Lee EJ, Timms P, and Polkinghorne A. 2015. Chlamydia pecorum infections in sheep and cattle: A common and under-recognised infectious disease with significant impact on animal health. The Veterinary Journal 206:252-260. http://dx.doi.org/10.1016/j.tvj1.2015.09.022

Walker E, Moore C, Shearer P, Jelocnik M, Bommana S, Timms P, and Polkinghorne A. 2016. Clinical, diagnostic and pathologic features of presumptive cases of Chlamydia pecorumassociated arthritis in Australian sheep flocks. BMC Veterinary Research 12:193. $10.1186 / \mathrm{s} 12917-016-0832-3$ Ndhlovu PD, Quinnell RJ, Watts CH, Chandiwana SK, and Anderson RM. 1997. Heterogeneities in the transmission of infectious agents: Implications for the design of control programs. Proceedings of the National Academy of Sciences 94:338-342. 
459 Yang R, Jacobson C, Gardner G, Carmichael I, Campbell AJD, and Ryan U. 2016. Longitudinal

460 prevalence and faecal shedding of Chlamydia pecorum in sheep (vol 201, pg 322, 2014).

461 Veterinary Journal 207:199-199. 10.1016/j.tvj1.2015.10.002 


\section{Figure legends}

463 Figure 1. Age dependent distribution of C. pecorum bacterial load and antibody titers (A) qPCR

464 load, lambs shedding high loads of $C$. pecorum DNA ( $\geq 1000$ copies/ $\mu 1)$ and CFT positive are

465 indicated as data points in red (B) CFT titer. From 2-6 months of age, lambs exhibited an overall

466 increase in mean chlamydial loads, peaking at 6 months of age (1128士6205). Mean CFT titres

467 were low at 2-4 months of age (9.5 \pm 4.5 and $8.3 \pm 2.8$ at 2 and 4 months), and then peaked from 6-

4688 months of age ( $29 \pm 27.8$ and $33.5 \pm 21$ at 6 and 8 months), followed by a slight decline in mean

469 titre at 10 months of age $(18.8 \pm 24)$, respectively.

470

471 Figure 2. Age dependent distribution of C. pecorum bacterial load at (A) Conjunctiva (B)

472 Rectum and (C) Vagina. Lambs shedding high loads of C. pecorum DNA ( $\geq 1000$ copies/ $\mu$ l) and

473 CFT positive are indicated as data points in red. The mean conjunctival bacterial loads trended

474 downward from 2-10 months of age (26.5 \pm 161.1 to $3.8 \pm 14.4$, respectively), in contrast, mean

475 bacterial loads increased from $2-6$ months of age $(16.3 \pm 115.3$ to $849.6 \pm 6143)$ at the rectal

476 sampling site, diminishing markedly thereafter $(0.8 \pm 2.3$ at 10 months). For female lambs the

477 greatest bacterial loads occurred from 6-10 months of age $(544.2 \pm 1538$ and $78.3 \pm 352.3$ at 6 and

47810 months) at the vaginal sampling site.

479

480

481

482

483

484 


\section{PeerJ}




\section{Table $\mathbf{1}$ (on next page)}

Characteristics of $C$. pecorum positivity of lambs $(n=76)$, as tested by qPCR at each sampling site (conjunctiva, rectum and vagina) and CFT. 
1 Table 1. Characteristics of $C$. pecorum positivity of lambs $(\mathrm{n}=76)$, as tested by $\mathrm{qPCR}$ at each

2 sampling site (conjunctiva, rectum and vagina) and CFT.

3

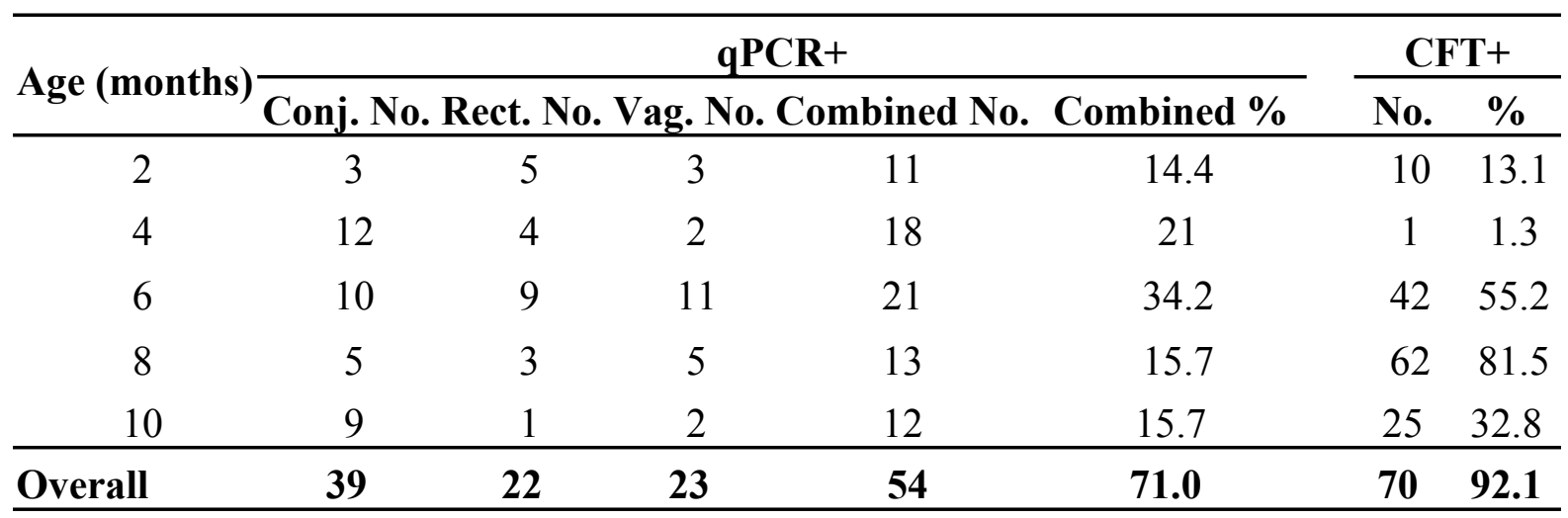




\section{Table 2 (on next page)}

Characteristics of qPCR CFT agreement, and the \% of lambs that cleared their infection by 10 months of age (as determined by a lamb having both a QPCR and CFT diagnosis across the sampling time points, but only being CFT positive by 10 months of age). 
1 Table 2. Characteristics of qPCR CFT agreement, and the \% of lambs that cleared their infection by 10 months of age (as determined

2 by a lamb having both a qPCR and CFT diagnosis across the sampling time points, but only being CFT positive by 10 months of age).

4

\begin{tabular}{ccccccc}
\hline Age (months) & $\begin{array}{c}\text { qPCR+CFT+ } \\
\text { No. }\end{array}$ & $\begin{array}{c}\text { qPCR+CFT- } \\
\text { No. }\end{array}$ & $\begin{array}{c}\text { qPCR-CFT+ } \\
\text { No. }\end{array}$ & $\begin{array}{c}\text { qPCR-CFT- } \\
\text { No. }\end{array}$ & $\begin{array}{c}\text { qPCR CFT } \\
\text { agreement \% }\end{array}$ & $\begin{array}{c}\text { Infection } \\
\text { clearance \% }\end{array}$ \\
\hline & & & & & & \\
2 & 2 & 6 & 8 & 60 & 81.5 & - \\
4 & 0 & 16 & 1 & 59 & 77.6 & - \\
6 & 22 & 4 & 23 & 27 & 64.4 & - \\
8 & 9 & 3 & 53 & 11 & 26.3 & - \\
10 & 5 & 7 & 20 & 44 & 64.4 & $\mathbf{7 1 . 0}$ \\
\hline Overall & $\mathbf{5 1}$ & $\mathbf{3}$ & $\mathbf{1 9}$ & $\mathbf{3 2 . 6}(\mathbf{n}=\mathbf{4 0})$ \\
\hline
\end{tabular}

5

6

7 


\section{Table 3 (on next page)}

First detection of $C$. pecorum in lambs at each sampling site (conjunctiva, rectum and vagina) by qPCR (infection). 
1 Table 3. First detection of C. pecorum in lambs at each sampling site (conjunctiva, rectum and

2 vagina) by qPCR (infection).

\begin{tabular}{cccccc}
\hline & \multicolumn{5}{c}{ qPCR+ (first detections) } \\
\cline { 2 - 6 } Age (months) & Conj. No. & Rect. No. & Vag. No. & Combined No. & Combined \% \\
\hline 2 & 3 & 5 & 3 & 11 & 14.4 \\
4 & 9 & 2 & 2 & 13 & 17.1 \\
6 & 10 & 5 & 10 & 25 & 32.8 \\
8 & 3 & 2 & 2 & 7 & 9.2 \\
10 & 7 & 1 & 2 & 10 & 13.1 \\
\hline
\end{tabular}

3

4 


\section{Table 4 (on next page)}

First detection of $C$. pecorum in lambs by CFT (antibodies) in relation to age.

Lambs with their first CFT detection were further categorised into (a) new CFT detection due to concurrent seroconversion i.e., matching qPCR detection, (b) new CFT detection due to delay in seroconversion as a result of previous qPCR positive infection, and (c) new CFT detection due to suspected previous infection that was missed due to bi-monthly sampling. 
1 Table 4. First detection of C. pecorum in lambs by CFT (antibodies) in relation to age. Lambs

2 with their first CFT detection were further categorised into (a) new CFT detection due to

3 concurrent seroconversion i.e., matching qPCR detection, (b) new CFT detection due to delay in

4 seroconversion as a result of previous qPCR positive infection, and (c) new CFT detection due to

5 suspected previous infection that was missed due to bi-monthly sampling.

6

\begin{tabular}{|c|c|c|c|c|c|}
\hline \multirow{2}{*}{$\begin{array}{c}\text { Age } \\
\text { (months) }\end{array}$} & \multicolumn{2}{|c|}{ CFT+ } & \multirow{2}{*}{$\begin{array}{c}\text { CFT+ due to } \\
\text { seroconversion }\end{array}$} & \multirow{2}{*}{$\begin{array}{l}\text { CFT+ due to lag in } \\
\text { seroconversion }\end{array}$} & \multirow{2}{*}{$\begin{array}{c}\text { CFT+ due to } \\
\text { suspected previous } \\
\text { exposure } \\
\end{array}$} \\
\hline & No. & $\%$ & & & \\
\hline 2 & 10 & 13.1 & 2 & 0 & 8 \\
\hline 4 & 0 & 0 & 0 & 0 & 0 \\
\hline 6 & 30 & 39.4 & 17 & 5 & 8 \\
\hline 8 & 20 & 26.3 & 5 & 9 & 6 \\
\hline 10 & 1 & 1.3 & 1 & 0 & 0 \\
\hline
\end{tabular}

7 


\section{Table 5 (on next page)}

Characteristics of $C$. pecorum bacterial load (qPCR load) and CFT antibody titers from 2 to 10 months of age. The qPCR load and CFT titers are expressed as mean and standard deviation. 
1 Table 5. Characteristics of C. pecorum bacterial load (qPCR load) and CFT antibody titers from 22 to 10 months of age. The qPCR load and CFT titers are expressed as mean and standard 3 deviation.

4

\begin{tabular}{cccccccccccc}
\hline \multirow{2}{*}{ Age (months) } & \multicolumn{2}{c}{ qPCR combined } & \multicolumn{2}{c}{ Conjunctiva } & \multicolumn{2}{c}{ Rectum } & \multicolumn{2}{c}{ Vagina } & \multicolumn{2}{c}{ CFT } \\
\cline { 2 - 11 } & Mean & SD & Mean & SD & Mean & SD & Mean & SD & Mean & SD \\
\hline 2 & 42.8 & 195.9 & 26.5 & 161.1 & 16.3 & 115.3 & 9.5 & 46.4 & 9.5 & 4.5 \\
4 & 31.6 & 169.4 & 13.5 & 91.1 & 19.03 & 144.7 & 0.9 & 4.1 & 8.3 & 2.8 \\
6 & 1128 & 6205 & 13.3 & 50.7 & 849.6 & 6143 & 544.2 & 1538 & 29 & 27.8 \\
8 & 44.8 & 192.1 & 9.6 & 61.3 & 3.4 & 18.4 & 64.3 & 253.8 & 33.5 & 21 \\
10 & 44.3 & 253.6 & 3.8 & 14.4 & 0.3 & 2.8 & 78.3 & 352.3 & 18.8 & 24 \\
\hline
\end{tabular}

5

6

7 


\section{Figure $\mathbf{1}$ (on next page)}

Age dependent distribution of $C$. pecorum bacterial load and antibody titers (A) qPCR load, lambs shedding high loads of $C$. pecorum DNA ( $\geq 1000$ copies/ $\mu$ ) and CFT positive are indicated as data points in red (B) CFT titer.

From 2-6 months of age, lambs exhibited an overall increase in mean chlamydial loads, peaking at 6 months of age $(1128 \pm 6205)$. Mean CFT titres were low at 2-4 months of age (9.5 \pm 4.5 and $8.3 \pm 2.8$ at 2 and 4 months), and then peaked from $6-8$ months of age $(29 \pm 27.8$ and $33.5 \pm 21$ at 6 and 8 months), followed by a slight decline in mean titre at 10 months of age $(18.8 \pm 24)$, respectively. 

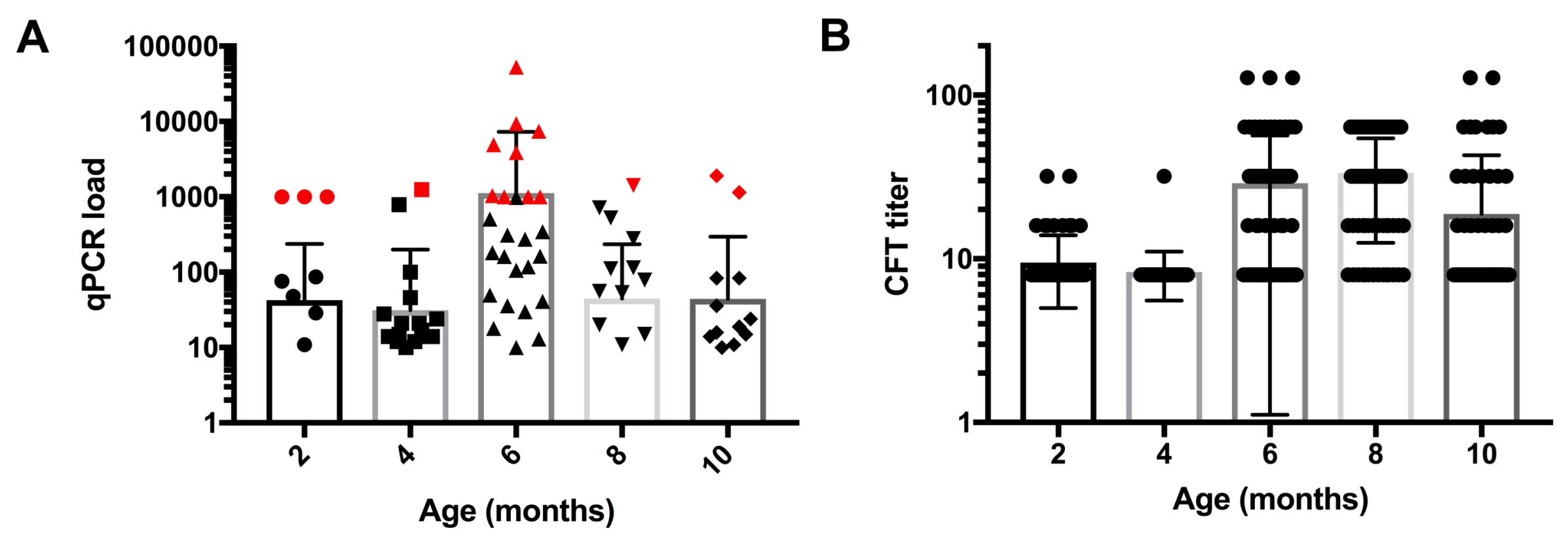
Figure 2 (on next page)

Age dependent distribution of $C$. pecorum bacterial load at (A) Conjunctiva (B) Rectum and (C) Vagina.

Lambs shedding high loads of $\mathrm{C}$. pecorum DNA ( $\geq 1000$ copies $/ \mu$ l) and CFT positive are indicated as data points in red. The mean conjunctival bacterial loads trended downward from 2-10 months of age (26.5 \pm 161.1 to $3.8 \pm 14.4$, respectively), in contrast, mean bacterial loads increased from $2-6$ months of age $(16.3 \pm 115.3$ to $849.6 \pm 6143)$ at the rectal sampling site, diminishing markedly thereafter $(0.8 \pm 2.3$ at 10 months). For female lambs the greatest bacterial loads occurred from $6-10$ months of age $(544.2 \pm 1538$ and $78.3 \pm 352.3$ at 6 and 10 months) at the vaginal sampling site. 


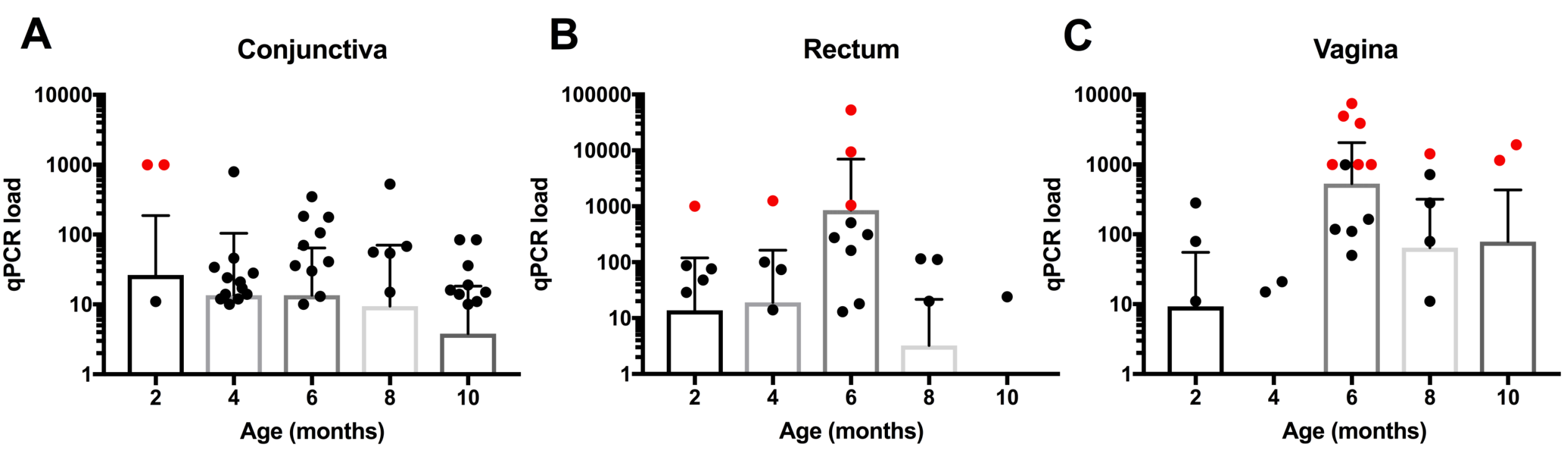

